\title{
GENERALIZATION OF THE CONCEPT OF SAMPLE SYMMETRY - FUZZY SYMMETRY, SYMMETROIDS, SIMILARITY
}

\author{
HANS-JOACHIM BUNGE* and INA NIELSEN \\ Department of Physical Metallurgy, Technical University of Clausthal, \\ Grosser Bruch 23, D-38678 Clausthal-Zellerfeld, Germany
}

(Received 1 November 1996)

The crystal orientation distribution function of polycrystalline materials, i.e. the texture, may exhibit internal symmetries due to symmetries of the production steps, or more generally, to the whole materials history. The "sharpness" of such symmetries can be quantified in terms of various symmetry parameters. If the symmetries of subsequent production processes are different, e.g. of sheet rolling and deep drawing, then these symmetries may still be recognized in the final texture. In the same way also similarities of textures and properties of different materials can be quantified. Symmetry parameters have many practical applications. Examples of that are the determination of rolling direction corrections, determination of the "plastic spin", estimation of coarse-grained materials, or finding the "correct" (symmetry adapted) axis system in a material.

Keywords: Sample symmetry; Diffuse symmetry; Fuzzyness; Symmetry degree; Symmetroids; Similarity of textures; Plastic spin

\section{INTRODUCTION}

The sample symmetry of an orientation distribution function (ODF) is illustrated in Fig. 1. The volume fraction of crystals in the orientation $g$ is equal to that of crystals in the symmetrically equivalent orientations $g \cdot g^{\mathrm{s}}$. Hence, sample symmetry is defined by the relationship

$$
f\left(g \cdot g^{\mathrm{s}}\right)=f(g) ; \quad g^{\mathrm{s}} \in G^{\mathrm{s}},
$$

* Corresponding author. Tel.: +495323 722 244. Fax: +49 5323722340 . 


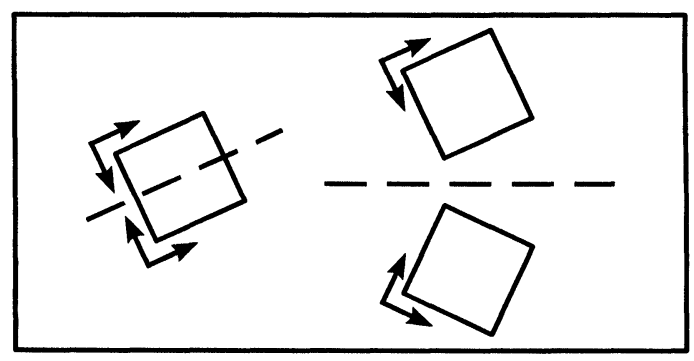
a) Crystal
b) Sample

Symmetry

FIGURE 1 Two symmetries in polycrystalline materials: (a) crystal symmetry holds for each individual single crystalline volume element; (b) sample symmetry holds for the volume fractions of crystallites in the two related orientations.

where $g^{\mathrm{s}}$ is an element of the (rotational) point symmetry group $G^{\mathrm{s}}$. Contrary to crystal symmetry which is fulfilled in each volume element individually, sample symmetry is defined only for the totality of all volume elements of crystallites in a statistical sense. Hence, it may be expected that it is less "sharp" than crystal symmetry. Furthermore, sample symmetry originates from the symmetry (or the symmetries) of all texture forming processes which have taken place in the history of the considered material, e.g. the orthorhombic symmetry of the sheetrolling process or the axial symmetry of wire drawing or many others. The symmetries of these processes can be more or less "sharp" so that the same must be assumed for the sample symmetry of a texture func. tion. Also different symmetries of different such processes may have been superposed, e.g. the orthorhombic symmetry of the sheet-rolling process and a monoclinic symmetry of a subsequent deep-drawing process. Hence, Eq. (1) will in general, only be an approximation.

\section{DEFINITION OF A SYMMETRY DEGREE - FUZZY SYMMETRY}

The "degree" of symmetry according to the symmetry element $g^{\mathrm{s}}$ can be judged by the difference function

$$
\Delta f\left(g, g^{\mathrm{s}}\right)=f(g)-f\left(g \cdot g^{\mathrm{s}}\right) .
$$


It may, however, be convenient to judge the degree of symmetry by a single-valued parameter. Such parameters can be defined in many different ways by various functionals $F$ of the difference function Eq. (2) written formally as

$$
F\left(g^{\mathrm{s}}\right)=F\left[\Delta f\left(g, g^{\mathrm{s}}\right)\right]
$$

As an example we may define the symmetry degree by the least-squares parameter $\sigma$ (i.e. a quadratic symmetry parameter)

$$
\sigma\left(g^{\mathrm{s}}\right)=1-\frac{\oint\left[f(g)-f\left(g \cdot g^{\mathrm{s}}\right)\right]^{2} \mathrm{~d} g}{2 \cdot \oint[f(\dot{g})]^{2} \mathrm{~d} g}
$$

which can also be expressed in the form

$$
\sigma\left(g^{\mathrm{s}}\right)=\frac{\oint f(g) \cdot f\left(g \cdot g^{\mathrm{s}}\right) \mathrm{d} g}{\oint[f(g)]^{2} \mathrm{~d} g},
$$

i.e. by the auto-correlation function of the texture function $f(g)$. This parameter may vary between

$$
0 \leq \sigma\left(g^{\mathrm{s}}\right) \leq 1,
$$

where

$$
\left.\begin{array}{rl}
=1 & \text { exact symmetry } \\
\sigma\left(g^{\mathrm{s}}\right)<1 & \text { "fuzzy" symmetry } \\
\rightarrow 0 & \text { no symmetry }
\end{array}\right\} \quad \text { symmetry degree } \sigma .
$$

Values of $\sigma<1$ correspond to some "unsharp" or "fuzzy" symmetry.

If the symmetry described by $g^{\mathrm{s}}$ is unsharp then $g^{\mathrm{s}}$ itself is also unsharp. Hence, it is necessary to consider the symmetry parameter $\sigma$, Eq. (4), also for other rotations $\Delta g$ instead of only the fixed symmetry elements $g^{\text {s }}$. Hence, we define analogous to Eq. (4)

$$
\sigma(\Delta g)=1-\frac{\oint[f(g)-f(g \cdot \Delta g)]^{2} \mathrm{~d} g}{2 \cdot \oint[f(g)]^{2} \mathrm{~d} g}=\frac{\oint f(g) \cdot f(g \cdot \Delta g) \mathrm{d} g}{\oint[f(g)]^{2} \mathrm{~d} g},
$$


which is the auto-correlation function of the texture function considered for variable $\Delta g$. Symmetry is then indicated by

$$
\operatorname{Max}[\sigma(\Delta g)] \text { at } \Delta g=g^{s} .
$$

Eq. (9) allows to find the symmetry elements $g^{\mathrm{s}}$ and the symmetry degree $\sigma\left(g^{`}\right)$ of these elements.

\section{SYMMETRY PARAMETERS FOR POLE FIGURES}

The symmetry parameters can also be defined for individual pole figures instead for the texture as a whole. In analogy to Eq. (8) we define the symmetry parameter for the pole figure $(h k l)$

$$
\begin{aligned}
\sigma_{(h k l)}(\Delta g) & =1-\frac{\oint\left[P_{(h k l)}(\vec{y})-P_{(h k l)}(\Delta g \cdot \vec{y})\right]^{2} \mathrm{~d} y}{2 \cdot \oint\left[P_{(h k l)}(\vec{y})\right]^{2} \mathrm{~d} y} \\
& =\frac{\oint P_{(h k /)}(\vec{y}) \cdot P_{(h k l)}(\Delta g \cdot \vec{y}) \mathrm{d} y}{\oint\left[P_{(h k /)}(\vec{y})\right]^{2} \mathrm{~d} y}
\end{aligned}
$$

with

$$
\vec{y}=\{\alpha, 3\}, \quad \mathrm{d} y=\sin \alpha \mathrm{d} \alpha \mathrm{d} \beta .
$$

The direction $\Delta g \cdot \vec{y}$ is that obtained from $\vec{y}$ by the rotation $\Delta g$. The symmetry in this pole figure can be judged analogous as in the case of the whole texture by Eq. (9).

\section{DEFINITION OF PARTIAL SYMMETRY - SYMMETROIDS}

A texture function may be composed of partial functions $f_{i}(g)$ with the volume fractions $V_{i}$ where each partial function may have its own sample symmetry group $G_{i}^{\mathrm{s}}$

$$
f(g)=\sum_{i=1}^{i_{\operatorname{mix}}} V_{i} \cdot f_{i}(g) ; \quad g_{i}^{\mathrm{s}} \in G_{i}^{\mathrm{s}} .
$$


In this case the function $\sigma(\Delta g)$, Eq. (8), will have local maxima

$$
\operatorname{Max}[\sigma(\Delta g)] \quad \text { at } \Delta g=g_{i}^{\mathrm{s}} \in G_{i}^{\mathrm{s}}
$$

in orientations $g_{i}^{s}$ which are the sum (unification set) of all symmetry elements of all the different symmetry groups $G_{i}^{\text {s }}$ of all partial texture functions $f_{i}(g)$. It must be mentioned that the values of $\sigma$ belonging to the symmetry elements $g_{\alpha}^{\mathrm{s}}$ of one of the partial functions need not necessarily be identical

$$
\sigma\left(g_{\alpha}^{\mathrm{s} 1}\right) \neq \sigma\left(g_{\alpha}^{\mathrm{s} 2}\right) ; \quad g_{\alpha}^{\mathrm{s} 1}, g_{\alpha}^{\mathrm{s} 2} \in G_{\alpha}^{\mathrm{s}} .
$$

This is due to the contributions of the other partial functions $f_{i}(g)$ with $i \neq \alpha$ which will, in general, be different in these orientations. It may even be that a particular symmetry element $g_{\alpha}^{s x}$ also belongs to the symmetry group $G_{\beta}^{\text {s }}$ of another partial function $f_{\beta}(g)$ which thus also contributes to the local maximum in this particular orientation. Hence, it seems consequent to consider all local maxima of the function $\sigma(\Delta g)$ as symmetry elements of some (hypothetical) partial texture function $f_{i}(g)$ in the sense of Eq. (9) independent of whether or not it is possible to actually split the function $f(g)$ into such partial function $f_{i}(g)$ as is assumed in Eq. (12). The orientations $g_{i}^{\text {s }}$ of these local maxima may be called symmetroids of the texture function $f(g)$. In the general case the symmetroids do not form a group. Rather they may be considered as the sum (or unification set) of the elements of several groups

$$
g_{i}^{\mathrm{s}} \in \tilde{G}^{\mathrm{s}}=G_{1}^{\mathrm{s}} \cup G_{2}^{\mathrm{s}} \cup \cdots \cup G_{m}^{\mathrm{s}} .
$$

\section{TEXTURE SIMILARITY}

We may compare two different textures $f_{1}(g)$ and $f_{2}(g)$. We may call them "similar" if the difference

$$
\Delta f(g)=f_{2}(g)-f_{1}(g)
$$

is small. As in the case of symmetry it may be convenient to have a single-valued parameter by which to judge "similarity". This may be a 
functional $F$ of the difference function $\Delta f(g)$ which we may write formally as

$$
F=F[\Delta f(g)]
$$

As an example we may define a least squares parameter $\sigma^{1,2}$ analogous to Eq. (4)

$$
\sigma^{1,2}=1-\frac{\oint\left[f_{2}(g)-f_{1}(g)\right]^{2} \mathrm{~d} g}{\oint\left[f_{1}(g)\right]^{2} \mathrm{~d} g+\oint\left[f_{2}(g)\right]^{2} \mathrm{~d} g}
$$

with this parameter similarity may be judged in the form

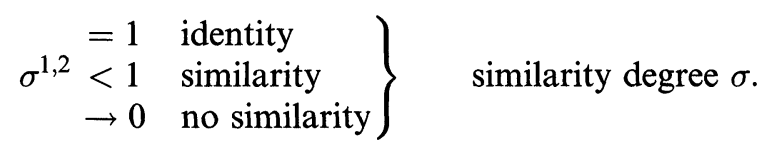

\section{Rotated Similarity}

If two textures $f_{1}(g)$ and $f_{2}(g)$ are not identical then some similarity may still exist if we consider either of them in its own particular sample coordinate system. Hence, we may define "rotated similarity" by

$$
\begin{aligned}
\sigma^{1,2}(\Delta g) & =1-\frac{\oint\left[f_{2}(g \cdot \Delta g)-f_{1}(g)\right]^{2} \mathrm{~d} g}{\oint\left[f_{1}(g)\right]^{2} \mathrm{~d} g+\oint\left[f_{2}(g)\right]^{2} \mathrm{~d} g} \\
& =\frac{2 \cdot \oint f_{2}(g \cdot \Delta g) \cdot f_{1}(g) \mathrm{d} g}{\oint\left[f_{1}(g)\right]^{2} \mathrm{~d} g+\oint\left[f_{2}(g)\right]^{2} \mathrm{~d} g}
\end{aligned}
$$

with this parameter rotated similarity may be judged in the form

$$
\operatorname{Max}\left[\sigma^{1,2}(\Delta g)\right] \text { at } \Delta g=\Delta g_{i}
$$

The rotations $\Delta g_{i}$ define the respective sample coordinate systems $K_{A_{1}}$ and $K_{A_{2}}$ with respect to which the two textures are similar (or even identical)

$$
K_{A_{2}}=\Delta g_{i} \cdot K_{A_{1}} .
$$

The function $\sigma^{1,2}(\Delta g)$ may have several local maxima. Then the two textures $f_{1}(g)$ and $f_{2}(g)$ are similar (possibly with different degree) in 
different relative orientations $\Delta g_{i}$. In analogy to Eq. (8) the similarity function $\sigma^{1,2}(\Delta g)$ is the intercorrelation function of the two texture functions $f_{1}(g)$ and $f_{2}(g)$. The similarity parameter $\sigma^{1,2}$, Eq. (18), referred to identical sample coordinate systems $K_{A_{1}}=K_{A_{2}}$ is, of course, contained in $\sigma^{1,2}(\Delta g)$

$$
\sigma^{1,2}=\sigma^{1,2}(\Delta g) \text { for } \Delta g=\{0,0,0\} .
$$

It is obvious that symmetry or fuzzy symmetry in the sense of Eqs. (8), (9) can be considered as "self-similarity" of a texture function $f(g)$.

\section{Similarity of Pole Figures}

Also the concept of similarity can be applied to individual pole figures. In analogy to Eq. (20) we define the similarity parameter for the $(h k l)$ pole figures of two textures

$$
\begin{aligned}
\sigma_{(h k l)}^{1,2}(\Delta g) & =1-\frac{\oint\left[P_{(h k l)}^{2}(\Delta g \cdot \vec{y})-P_{(h k l)}^{1}(\vec{y})\right]^{2} \mathrm{~d} y}{\oint\left[P_{(h k l)}^{2}(\vec{y})\right]^{2} \mathrm{~d} y+\oint\left[P_{(h k l)}^{1}(\vec{y})\right]^{2} \mathrm{~d} y} \\
& =\frac{2 \cdot \oint P_{(h k l)}^{2}(\Delta g \cdot \vec{y}) \cdot P_{(h k l)}^{1}(\vec{y}) \mathrm{d} y}{\oint\left[P_{(h k l)}^{2}(\vec{y})\right]^{2} \mathrm{~d} y+\oint\left[P_{(h k l)}^{1}(\vec{y})\right]^{2} \mathrm{~d} y} .
\end{aligned}
$$

With the definition of Eq. (24) similarity of the two pole figures may then be judged according to Eq. (21).

\section{Similarity of Non-Equivalent Pole Figures}

In certain cases it may even be meaningful to compare pole figures of different lattice planes $(h k l)\left(h^{\prime} k^{\prime} l^{\prime}\right)$ with each other. Particularly we consider this case for different pole figures of the same texture. Then a rotation $\Delta g$ between the two will not be meaningful. Hence, for this case we define a similarity parameter in analogy to Eq. (18)

$$
\sigma_{\left(h k l, h^{\prime} k^{\prime} l^{\prime}\right)}^{1,2}=1-\frac{\oint\left[P_{(h k l)}(\vec{y})-P_{\left(h^{\prime} k^{\prime} l^{\prime}\right)}(\vec{y})\right]^{2} \mathrm{~d} y}{\oint\left[P_{(h k l)}(\vec{y})\right]^{2} \mathrm{~d} y+\oint\left[P_{\left(h^{\prime} k^{\prime} l^{\prime}\right)}(\vec{y})\right]^{2} \mathrm{~d} y} .
$$


Similarity of these two pole figures is then judged in analogy to Eq. (19). This parameter is important for ODF analysis from pole figures. If some of the "input pole figures" used in ODF analysis are "too similar" then they do not increase the independent input information needed for ODF-calculation.

\section{SYMMETRY PARAMETERS BASED ON SERIES EXPANSION}

We represent the texture function $f(g)$ by a series expansion in the form

$$
f(g)=\sum_{\lambda=0}^{L} \sum_{m=-\lambda}^{+\lambda} \sum_{n=-\lambda}^{+\lambda} C_{\lambda}^{m n} T_{\lambda}^{m n}(g) .
$$

Using the addition theorem of the functions $T_{\lambda}^{m n}(g)$ the symmetry function $\sigma(\Delta g)$, Eq. (8) can be written in the form (see e.g. Bunge, 1982)

$$
\sigma(\Delta g)=\sum_{\lambda=0}^{L} \sum_{m=-\lambda}^{+\lambda} \sum_{n=-\lambda}^{+\lambda} C_{\lambda}^{\prime m n} T_{\lambda}^{m n}(\Delta g)
$$

with the coefficients

$$
C_{\lambda}^{\prime m n}=\frac{\sum_{s=-\lambda}^{+\lambda} C_{\lambda}^{s m} \cdot C_{\lambda}^{s n}}{\sum_{\lambda m n}\left[C_{\lambda}^{m n}\right]^{2}} .
$$

Eq. (28) shows again that $\sigma(\Delta g)$ is a quadratic symmetry parameter.

\section{Rotation Axes in $z$-Direction}

Symmetry axes of $r$ th order in $z$-direction of the sample coordinate system $K_{A}$ are represented in terms of the "selection rule" (see e.g. Bunge, 1982)

$$
C_{\lambda}^{m n}=0 \quad \text { for } n \neq r \cdot n^{\prime}
$$


Hence, a linear symmetry parameter can be defined on this basis. We put

$$
\varphi^{r}=\sum_{\lambda=0}^{L} \sum_{m=-\lambda}^{+\lambda} \sum_{n=-\lambda}^{+\lambda}\left|C_{\lambda}^{m n}\right|, \quad n \neq r \cdot n^{\prime} .
$$

Symmetry is indicated by this parameter by the condition

$$
\left.\begin{array}{rlr} 
& =0 & \text { exact symmetry } \\
\varphi^{r} & =\text { low } & \text { fuzzy symmetry } \\
& =\text { high } & \text { no symmetry }
\end{array}\right\} \quad \text { symmetry degree } \varphi^{r} .
$$

It must be mentioned that in the sample symmetry rotation axes of all orders $r$ may occur, particularly also those for $r=\infty$, i.e. axial symmetry. In fact, these latter ones are particularly interesting.

The parameter $\varphi^{r}$, Eq. (30), can also be defined in any rotated sample coordinate system according to Eq. (22). In the sample coordinate system rotated through $\Delta g$ the texture function $f(g)$ may be written in the form

$$
f^{\prime}(g)=f(g \cdot \Delta g)=\sum_{\lambda=0}^{L} \sum_{m=-\lambda}^{+\lambda} \sum_{n=-\lambda}^{+\lambda} C_{\lambda}^{\prime m n}(\Delta g) \cdot T_{\lambda}^{m n}(g)
$$

with the "transformed" texture coefficients (see e.g. Bunge, 1982)

$$
C_{\lambda}^{\prime m n}=\sum_{s=-\lambda}^{+\lambda} C_{\lambda}^{m s} \cdot T_{\lambda}^{s n}(\Delta g)
$$

Hence, we define the (linear) symmetry parameter function

$$
\varphi^{r}(\Delta g)=\sum_{\lambda=0}^{L} \sum_{m=-\lambda}^{+\lambda} \sum_{n=-\lambda}^{+\lambda}\left|C_{\lambda}^{\prime m n}(\Delta g)\right|
$$

which indicates symmetry in the $z^{\prime}$-axis of the rotated sample coordinate system

$$
K_{A}^{\prime}=\Delta g \cdot K_{A}
$$


according to

$$
\left.\begin{array}{rlr}
\varphi^{r}(\Delta g) & =0 & \text { exact symmetry } \\
& =\text { high } & \text { nuzzy symmetry } \\
\text { no symmetry }
\end{array}\right\} \quad \text { at } \Delta g \rightarrow\{\alpha, \beta\} .
$$

Since the parameter $\varphi^{r}(\Delta g)$ "sees" only symmetry axes in $z^{\prime}$-direction it is sufficient to consider it only for rotations of the form

$$
\Delta g=\left\{\Delta \varphi_{1}, \Delta \phi, 0\right\} .
$$

The angles $\Delta \varphi_{1}$ and $\Delta \phi$ may then be plotted as pole figure angles $\{\alpha \beta\}$ in the original sample coordinate system $K_{A}$ in the form

$$
\alpha=\Delta \phi, \quad \beta=\Delta \varphi_{1}-90^{\circ} .
$$

Then the position of axes of order $r$ can be seen directly in the pole figure of the original sample coordinate system $K_{A}$.

Local minima of the function $\varphi^{r}(\Delta g)$ indicate symmetries and more generally symmetroids according to Eq. (15).

The criterion $\varphi^{r}(\Delta g)$, Eq. (34), is defined by absolute values of the coefficients $C_{\lambda}^{m n}$ in contrast to the criterion $\sigma(\Delta g)$, Eq. (8), which is defined by the square of the difference function. Hence, the criterion $\varphi^{r}(\Delta g)$ exhibits apexes in the symmetry positions whereas $\sigma(\Delta g)$ goes through these positions with horizontal tangent.

\section{Mirror Plane Perpendicular to the $y$-Axis}

Taking crystal symmetry into account the series expansion Eq. (26) can be written in the form (see e.g. Bunge, 1982)

$$
f(g)=\sum_{\lambda=0}^{L} \sum_{\mu=1}^{M(\lambda)} \sum_{n=-\lambda}^{+\lambda} C_{\lambda}^{\mu n} \dot{T}_{\lambda}^{\mu n}(g) .
$$

With this form of the series expansion, any pole figure can be written in the form

$$
P_{(h k l)}(\alpha \beta)=\sum_{\lambda=0}^{L} \sum_{n=-\lambda}^{+\lambda}\left[\sum_{\mu=1}^{M(\lambda)} \frac{4 \pi}{2 \lambda+1} C_{\lambda}^{\mu n} \cdot \dot{k}_{\lambda}^{* \mu}(h k l)\right] \cdot k_{\lambda}^{n}(\alpha \beta) .
$$


This function possesses mirror symmetry perpendicular to the $y$-axis of the sample coordinate system $K_{A}$ if the expression in brackets is real. If we assume (for simplicity) that the functions $\dot{k}_{\lambda}^{* \mu}(h k l)$ are real (as it is usual in cubic crystal symmetry) then this requires

$$
C_{\lambda}^{\mu n}=C_{\lambda}^{* \mu n} \quad(\text { real })
$$

Hence, a symmetry parameter indicating mirror symmetry in all pole figures perpendicular to the sample direction $y$ can be defined by

$$
\varphi^{M}=\sum_{\lambda=0}^{L} \sum_{\mu=1}^{M(\lambda)} \sum_{n=-\lambda}^{+\lambda}\left|I C_{\lambda}^{\mu n}\right|
$$

with

$$
I C_{\lambda}^{\mu n}=\frac{1}{2}\left[C_{\lambda}^{\mu n}-C_{\lambda}^{* \mu n}\right] \quad \text { (imaginary part). }
$$

We assume further that the pole figures are centrosymmetric which is the case for

$$
\lambda=2 \lambda^{\prime}, \quad \text { even }
$$

Then a mirror plane perpendicular to the $y$-direction corresponds to a two-fold axis in this direction. Hence, the parameter $\varphi^{M}$ indicates this symmetry by the condition

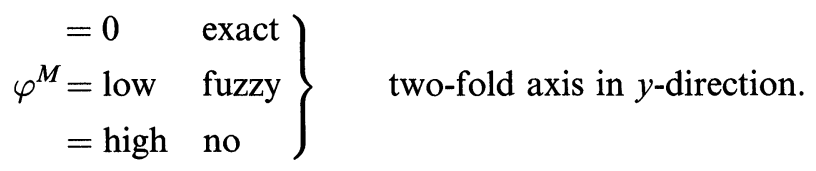

This parameter is thus equivalent (though not numerically identical with) $\varphi^{r=2}$.

Again we consider this parameter in all rotated sample coordinate systems $K_{A}^{\prime}$, Eq. (35)

$$
\varphi^{M}(\Delta g)=\sum_{\lambda=0}^{L} \sum_{\mu=1}^{M(\lambda)} \sum_{n=-\lambda}^{+\lambda}\left|I C_{\lambda}^{\prime \mu n}(\Delta g)\right|
$$


with

$$
C_{\lambda}^{\prime \mu n}(\Delta g)=\sum_{s=-\lambda}^{+\lambda} C_{\lambda}^{\mu s} \cdot T_{\lambda}^{s n}(\Delta g)
$$

A two-fold axis in $y^{\prime}$-direction is thus indicated by

$$
\left.\begin{array}{rlr}
\varphi^{M}(\Delta g) & =0 & \text { exact } \\
& =\text { high } & \text { fuzzy } \\
\text { no }
\end{array}\right\} \quad \text { at } \Delta g \rightarrow\{\alpha, \beta\}
$$

The $y^{\prime}$-direction can be brought into all positions relative to the original sample coordinate system $K_{A}$ by rotations of the form

$$
\Delta g=\left\{\Delta \varphi_{1}, \Delta \phi, 0\right\}
$$

These angles can be plotted as pole figure angles $\{\alpha \beta\}$ in $K_{A}$ in the form

$$
\alpha=90^{\circ}-\Delta \phi, \quad \beta=90^{\circ}+\Delta \varphi_{1} .
$$

In this representation the criterion $\varphi^{M}(\Delta g)$ indicates a two-fold rotation axis in the direction $\{\alpha \beta\}$. More generally, local minima of this parameter indicate symmetroids according to Eq. (15).

\section{Symmetry Parameters for Pole Figures}

A symmetry parameter similar to Eq. (30) can also be defined on the basis of the pole figure coefficients $F_{\lambda}^{n}(h k l)$ instead of the texture coefficients $C_{\lambda}^{m n}$. We define

$$
\varphi_{(h k l)}^{r}=\sum_{\lambda=0}^{L} \sum_{n=-\lambda}^{+\lambda}\left|F_{\lambda}^{n}(h k l)\right|, \quad n \neq r \cdot n^{\prime}
$$

with

$$
F_{\lambda}^{n}(h k l)=\frac{4 \pi}{2 \lambda+1} \sum_{m=-\lambda}^{+\lambda} C_{\lambda}^{m n} \cdot k_{\lambda}^{* m}(h k l)
$$


Also this symmetry parameter has to be considered with respect to a rotated sample coordinate system $K_{A}^{\prime}$ defined by Eq. (35)

$$
\varphi_{(h k l)}^{r}(\Delta g)=\sum_{\lambda=0}^{L} \sum_{n=-\lambda}^{+\lambda}\left|F_{\lambda}^{\prime n}(h k l, \Delta g)\right|, \quad n \neq r \cdot n^{\prime}
$$

with

$$
F_{\lambda}^{\prime n}(h k l, \Delta g)=\sum_{s=-\lambda}^{+\lambda} F_{\lambda}^{s}(h k l) \cdot T_{\lambda}^{s n}(\Delta g) .
$$

Estimation of symmetry with this parameter is analogous to Eq. (36).

Also the symmetry parameter Eq. (42) can be defined for an individual pole figure. We put

$$
\varphi_{(h k l)}^{M}=\sum_{\lambda=0}^{L} \sum_{n=-\lambda}^{+\lambda}\left|I F_{\lambda}^{n}(h k l)\right|
$$

with

$$
\left|I F_{\lambda}^{n}(h k l)\right|=\frac{1}{2}\left[F_{\lambda}^{n}(h k l)-F_{\lambda}^{* n}(h k l)\right] .
$$

According to Eq. (45) this parameter tests the existence of a mirror plane perpendicular to the $y$-direction or with Eq. (44), a two-fold axis in this direction. Also this parameter can be considered in any rotated sample coordinate system. We define in analogy to Eq. (46)

$$
\varphi_{(h k l)}^{M}(\Delta g)=\sum_{\lambda=0}^{L} \sum_{n=-\lambda}^{+\lambda}\left|I F_{\lambda}^{\prime n}(h k l, \Delta g)\right|
$$

with $F^{\prime}$ related to $F$ according to Eq. (54). The existence of a mirror plane or two-fold axis is then estimated according to Eq. (48).

The criterion $\varphi_{h k l}^{M}$ is particularly obvious because it contains the terms $\sin n \beta$ in the series expansion of a pole figure. These terms change their sign when going through $\beta=0$. Hence, these are the terms which break the mirror symmetry in $\beta=0$. 


\section{SIMILARITY PARAMETERS BASED ON SERIES EXPANSION}

In complete analogy to Eqs. (27), (28) also the similarity function Eq. (20) can be expressed in terms of the series expansion, Eq. (26), applied to both textures $f_{1}(g)$ and $f_{2}(g)$. Thus we obtain

$$
\sigma^{1,2}(\Delta g)=\sum_{\lambda=0}^{L} \sum_{m=-\lambda}^{+\lambda} \sum_{n=-\lambda}^{+\lambda} C_{\lambda}^{\prime m n} \cdot T_{\lambda}^{m n}(\Delta g)
$$

with the coefficients

$$
C_{\lambda}^{\prime m n}=\frac{2 \cdot \sum_{s=-\lambda}^{+\lambda} C_{\lambda}^{s m}(2) \cdot C_{\lambda}^{s n}(1)}{\sum_{\lambda m n}\left\{\left[C_{\lambda}^{m n}(1)\right]^{2}+\left[C_{\lambda}^{m n}(2)\right]^{2}\right\}} .
$$

Again Eq. (59) shows the quadratic nature of this similarity parameter.

A linear similarity paramater can be defined in the form

$$
\varphi^{1,2}=\sum_{\lambda=0}^{L} \sum_{m=-\lambda}^{+\lambda} \sum_{n=-\lambda}^{+\lambda}\left|C_{\lambda}^{m n}(2)-C_{\lambda}^{m n}(1)\right| .
$$

Similarity of the two textures can be judged by this parameter in the form

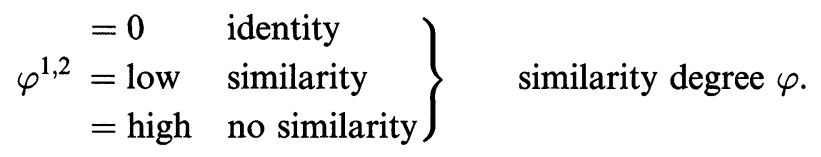

Accordingly, also rotated similarity can be expressed in the form

$$
\varphi^{1,2}(\Delta g)=\sum_{\lambda=0}^{L} \sum_{m=-\lambda}^{+\lambda} \sum_{n=-\lambda}^{+\lambda}\left|C_{\lambda}^{\prime m n}(2, \Delta g)-C_{\lambda}^{m n}(1)\right|
$$

with $C_{\lambda}^{\prime m n}(2, \Delta g)$ according to Eq. (33). Rotated similarity can be judged in the form

$$
\operatorname{Min}\left[\varphi^{1,2}(\Delta g)\right] \text { at } \Delta g=\Delta g_{i}
$$


Also pole figure similarity parameters can be defined in terms of the series expansion coefficients. In analogy to Eq. (60) we define

$$
\varphi_{(h k l)}^{1,2}=\sum_{\lambda=0}^{L} \sum_{n=-\lambda}^{+\lambda}\left|F_{\lambda}^{n}(h k l, 2)-F_{\lambda}^{n}(h k l, 1)\right|,
$$

which is to be judged according to Eq. (61). It may also be meaningful to consider different pole figures $(h k l)$ and $\left(h^{\prime} k^{\prime} l^{\prime}\right)$. Then the parameter

$$
\varphi_{\left(h k l, h^{\prime} k^{\prime} l^{\prime}\right)}=\sum_{\lambda=0}^{L} \sum_{n=-\lambda}^{+\lambda}\left|F_{\lambda}^{n}(h k l)-F_{\lambda}^{n}\left(h^{\prime} k^{\prime} l^{\prime}\right)\right|
$$

may be helpful. According to this parameter it can be estimated how much "new" information the pole figure $\left(h^{\prime} k^{\prime} l^{\prime}\right)$ contributes to ODF analysis additional to the pole figure $(h k l)$.

\section{SYMMETROIDS DUE TO TEXTURE FORMATION PROCESSES}

Textures are formed by solid state processes of any kind. These processes modify an initial texture into the final texture. If several such processes are acting one after the other, the final texture is the result of the superposition of all these processes

$$
f^{\text {end }}(g)=M_{n} \otimes \cdots \otimes M_{2} \otimes M_{1} \otimes f^{\text {initial }}(g) .
$$

Each of these processes may have its own symmetry which is the symmetry of the "modifyer operators" $M_{i}$. Strictly speaking, the final texture can only have a sample symmetry which is the lowest subsymmetry of all processes, i.e. of all modifyers $M_{i}$. The individual symmetries may, however, still be seen in the final texture in the form of symmetroids. This is particularly interesting in geological sciences, where a final texture is measured in order to get information about a sequence of "modifyers" which have contributed to it during the materials history. 


\section{SOME EXAMPLES}

In the following some examples are considered which illustrate the practical application of the defined parameters and the conclusions which can be drawn from them.

The first example is the rolling texture of a cold rolled copper sheet. Fig. 2(a) shows the (111) pole figure. Intentionally, the rolling direction was not well-aligned in the sample holder. Rather it deviates by the angle $\beta_{0}$ from the zero position. Besides the (111) pole figure also (200) and (220) were measured all in steps of $\Delta \alpha=5^{\circ}, \Delta \beta=3.6^{\circ}$. From these pole figures the ODF was calculated using the assumption of triclinic sample

a)

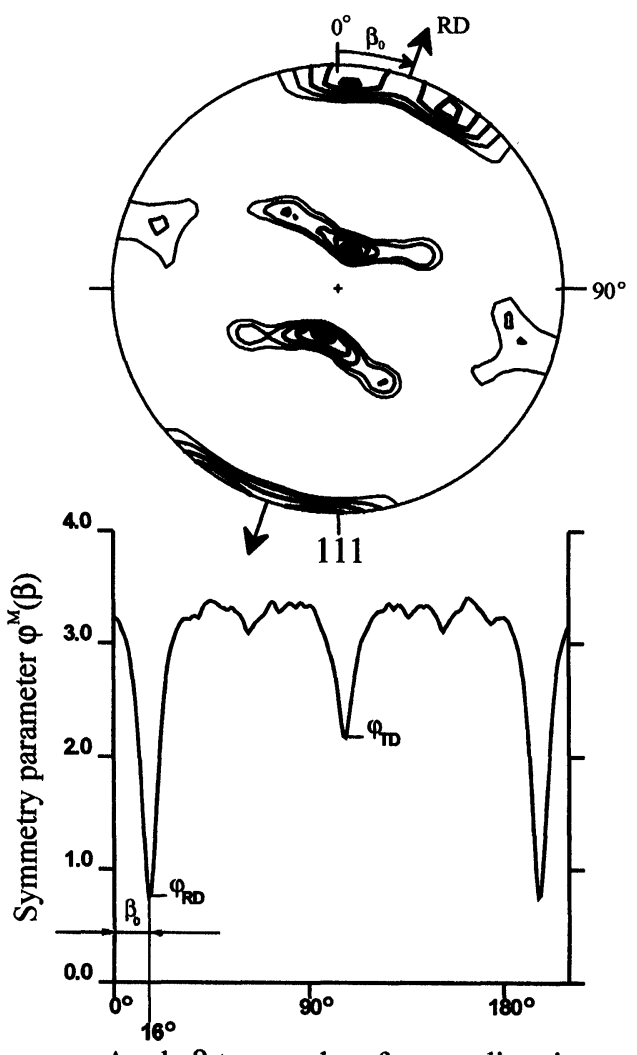

b)

Angle $\beta$ to sample reference direction

FIGURE 2 Symmetry analysis of the rolling texture of copper: (a) (111) pole figure (recalculated from ODF data); (b) symmetry parameter $\varphi^{M}(\beta)$ plotted in the rolling plane. 
symmetry. Series expansion degree was $L=22$. From the series expansion coefficients $C_{\lambda}^{\mu n}$ the symmetry parameter $\varphi^{M}(\Delta g)$ according to Eq. (46) was calculated. It is plotted in Fig. 2(b) as a function of the angle $\beta=90^{\circ}+\Delta \varphi_{1}$, Eq. (50). This curve shows a very sharp minimum at $\beta_{0}=16^{\circ}$ which represents the alignment correction of the rolling direction. The accuracy of the value $\beta_{0}$ is much higher than with the conventionally used alignment corrections.

The curve Fig. 2(b) also shows a second minimum at $\beta_{0}+90^{\circ}$ which corresponds to the transverse direction. This shows that the sample symmetry is orthorhombic. The value of $\varphi^{M}$ (TD) is higher than $\varphi^{M}(\mathrm{RD})$. This illustrates that the two mirror planes in RD and TD have different symmetry degrees. The one in TD is more "fuzzy" than the one in RD.

In Fig. 3 the analogous situation is illustrated for a recrystallized copper sheet. The pole figure (200) is shown in this case. The parameter $\varphi^{M}(\beta)$ is plotted in Fig. 3(b). Again the alignment correction angle $\beta_{0}=59^{\circ}$ can be obtained from this diagram with good accuracy. Besides the minimum in $\mathrm{RD}$ there are now other ones in $\mathrm{RD} \pm 90^{\circ}$ and $\mathrm{RD} \pm 45^{\circ}$. This time the values at RD and TD are very close to each other, there is, however, a slightly higher deviation in $\mathrm{RD} \pm 45^{\circ}$. This shows that the sample symmetry is now close to tetragonal but a slight right-left asymmetry from the rolling direction can be seen (Nielsen and Bunge, 1996).

A third example concerns a coarse grained material. The samples were A1 $99.5 \%$ purity, deformed respectively $50 \%$ and $70 \%$. After that they were annealed $24 \mathrm{~h}$ at $400^{\circ}$. The resulting texture is a weak cube texture. As a result of that the pole figures are rather "spiky". Nevertheless, Fig. 4(a), i.e. for the $70 \%$ material, shows definitely the four minima similar to Fig. 3 indicating the tetragonal sample symmetry characteristic for the cube texture. The situation in Fig. 4(b) is even more "fuzzy" but still the four minima can be recognized.

Another example is shown in Fig. 5. The material is a ferritic steel St14Q5. Texture measurement, this time, was done with the oblique section method (see e.g. Welch, 1980). In this technique oblique sections through the sheet are used which have ideally their normal direction under equal angles (i.e. $54.7^{\circ}$ ) to the sheet directions ND, RD, TD. These sample cutting angles as well as the alignment of the sample in the sample holder may, however, deviate from their ideal values. In this case the 
a)
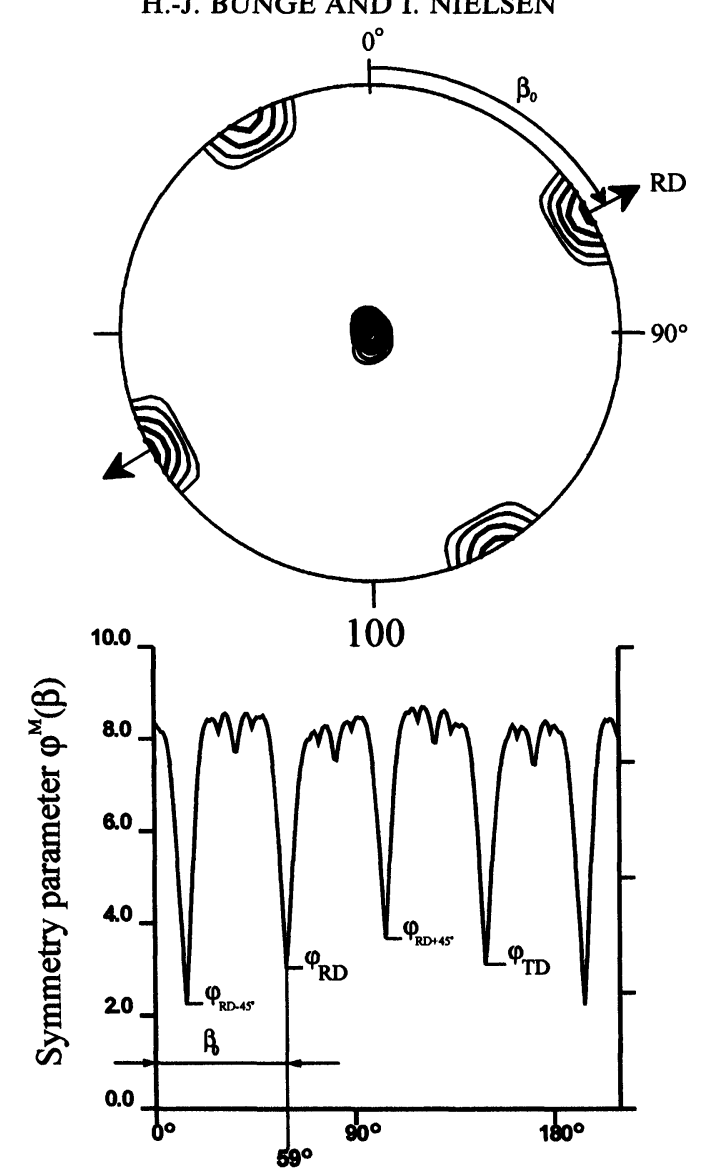

b)

Angle $\beta$ to sample reference direction

FIGURE 3 Symmetry analysis of the cube texture of copper: (a) (200) pole figure (recalculated from ODF data); (b) symmetry parameter $\varphi^{M}(\beta)$ plotted in the rolling plane.

"back-transformation" of the measured pole figures to the sheet coordinate system ND, TD, RD may be incorrect. Hence, ODF analysis was carried out in the "measuring coordinates" assuming triclinic sample symmetry. Then the symmetry parameter $\varphi^{r=2}(\Delta g)$, Eq. (34), was determined, this time in the whole two-dimensional area, Eq. (38). The result is plotted in the middle-left in Fig. 5. One sees very clearly the minima corresponding to the three orthorhombic axes i.e. ND, TD, RD. It is also seen that one of them is much less "fuzzy" than the other two. This is the normal direction. Also a broad "fuzzy-band" can be seen in the 
rolling plane. From this diagram the transformation angles for transformation into ND, TD, RD can easily be obtained. The transformation was carried out in the middle-right diagram and also in the pole figures (lower line in Fig. 5). This example shows, on the one hand, the most reliable evaluation method for oblique section measurement. On the other hand it shows the much sharper nature of the symmetry axis in ND and of the mirror plane in the rolling plane compared with the symmetry elements in the rolling plane.

(a)
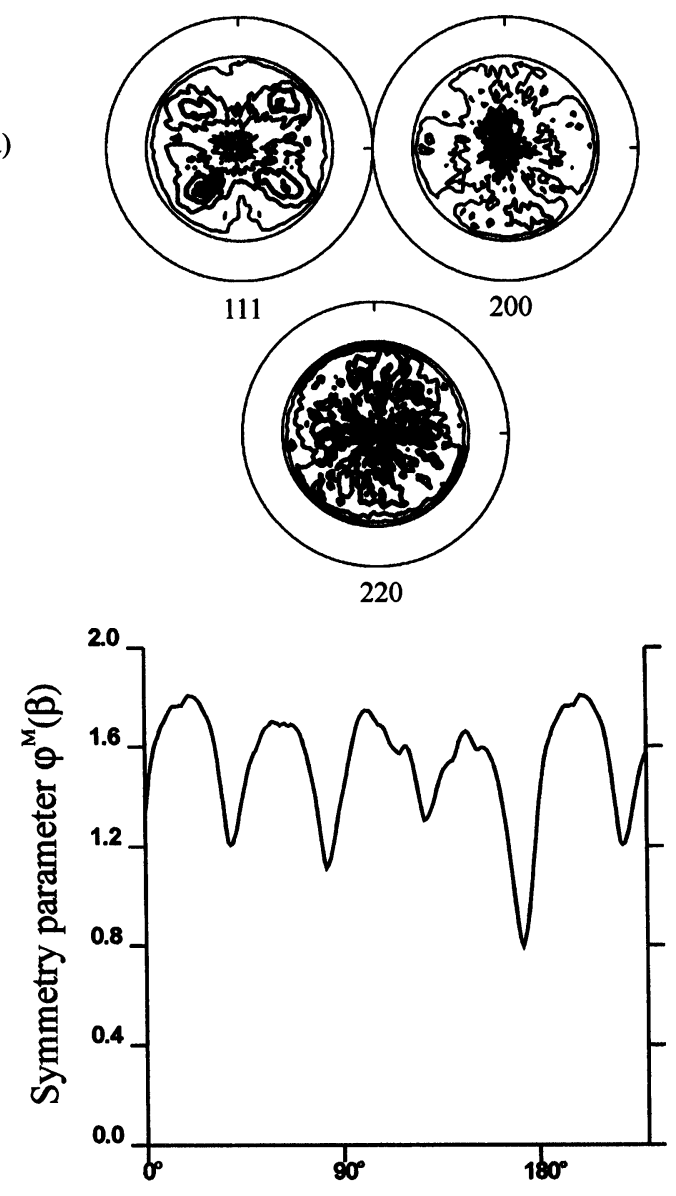

Angle $\beta$ to sample reference direction 
(b)
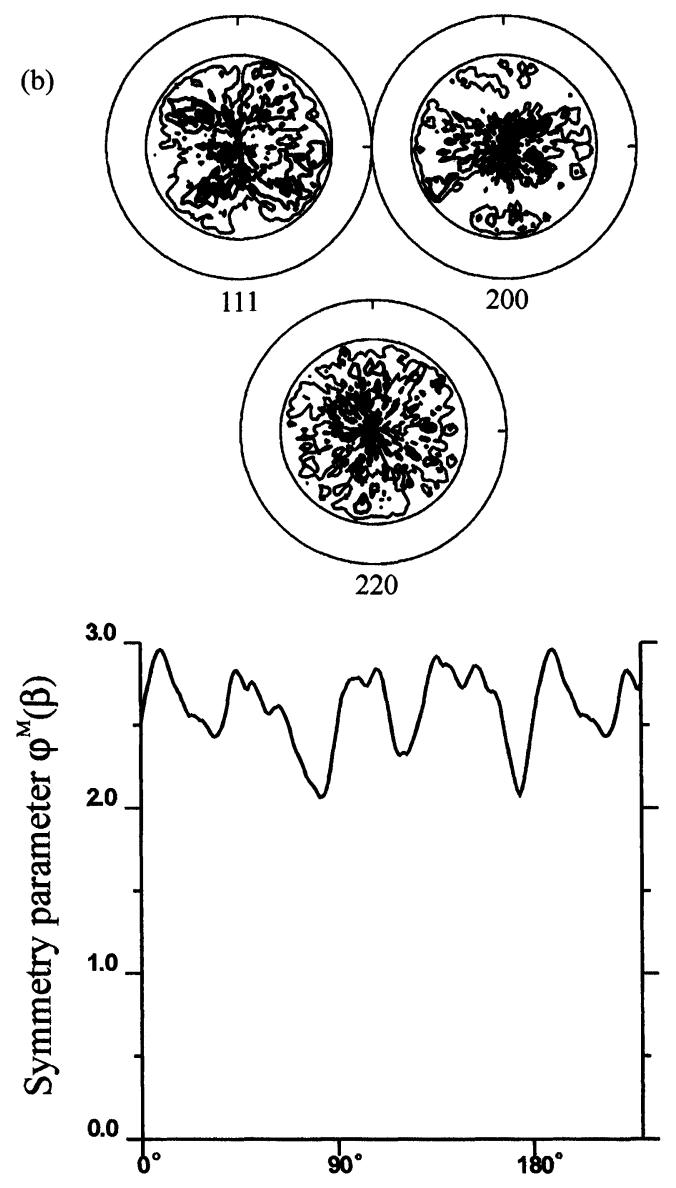

Angle $\beta$ to sample reference direction

FIGURE 4 Symmetry analysis of coarse grained A 199.5 , recrystallized $24 \mathrm{~h}$ at $400^{\circ} \mathrm{C}$. Three experimental pole figures and the symmetry parameter $\varphi^{M}(\beta)$ in the rolling plane: (a) after $70 \%$ cold rolling; (b) after $50 \%$ cold rolling.

In Fig. 6 the same as in the preceeding example is shown. Here the material was an austenitic steel $\mathrm{X} 5 \mathrm{CrNi} 1810$ in the cold rolled state. From the oblique section pole figures, upper line, ODF was calculated and from the texture coefficients again the $\varphi^{r=2}(\Delta g)$, middle-left. This gives very clearly the transformation angles to the sheet coordinate system ND, TD, RD which was carried out in middle-right. Finally, the 


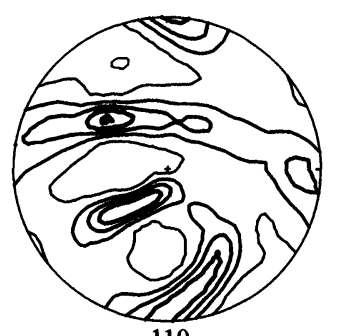

110
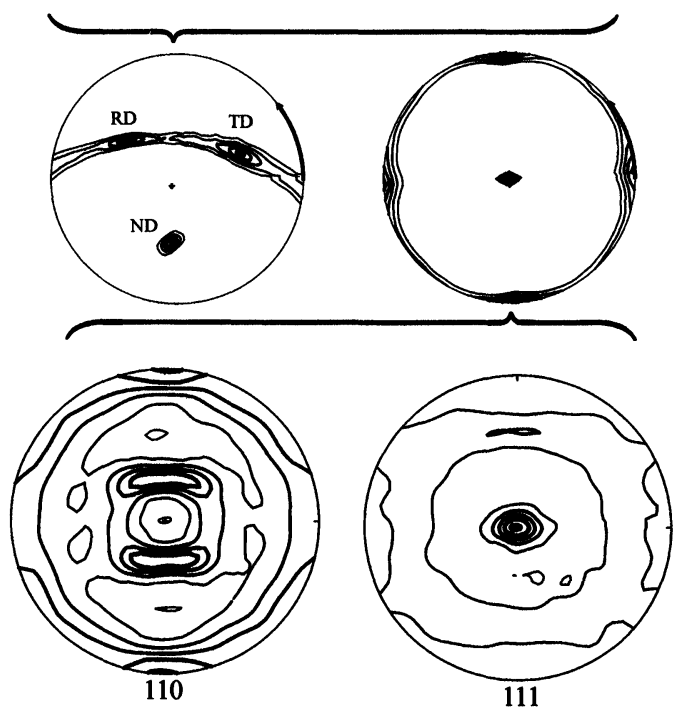

FIGURE 5 Symmetry analysis of a deep drawing steel St14Q5 from oblique section pole figure measurements: upper row: two oblique section pole figures (recalculated from ODF data with triclinic sample symmetry); middle row: the symmetry parameter $\varphi^{r=2}(\Delta g)$ plotted as pole figures in the oblique coordinate system and after transformation to RD, TD, ND; lower row: the pole figures transformed to RD, TD, ND.

lower part of this figure shows the pole figures represented in the conventional coordinate system ND, TD, RD.

As a further example, a series of aluminum samples was considered. They were recrystallized with the starting texture being essentially the cube texture. Then samples were cut at different angles $\tau$ to the original rolling direction and were stretched $20 \%$ in the indicated direction SD in Fig. 7. The (111) pole figures of the stretched samples are shown in Fig. 8. Besides (111) also (200) and (220) were measured from which ODF could be obtained assuming triclinic sample symmetry. From the 


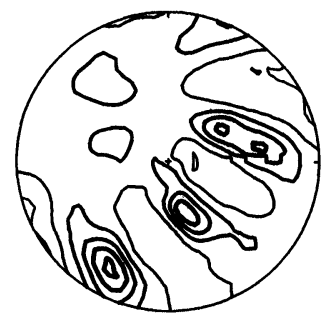

111

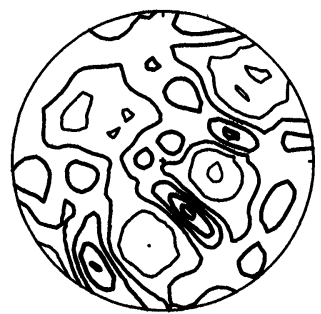

100

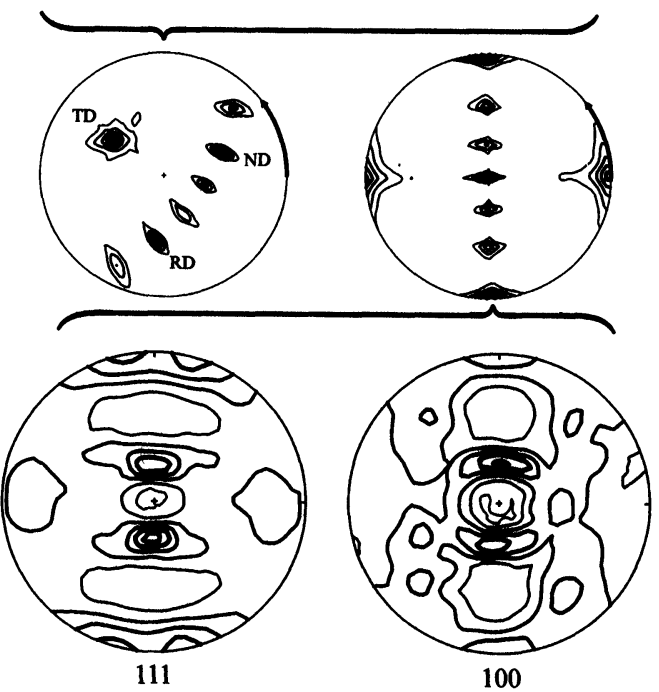

FIGURE 6 Symmetry analysis of an austenitic steel X5CrNi1810 from oblique section pole figure measurement analogous to Fig. 5 .

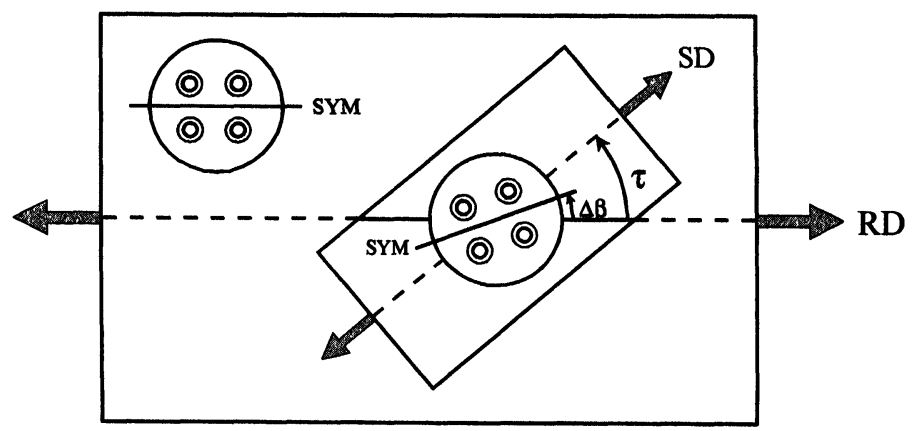

FIGURE 7 Tensile samples taken under different angles $\tau$ from sheet material indicating the angle $\Delta \beta$ of the symmetry plane after deformation. 

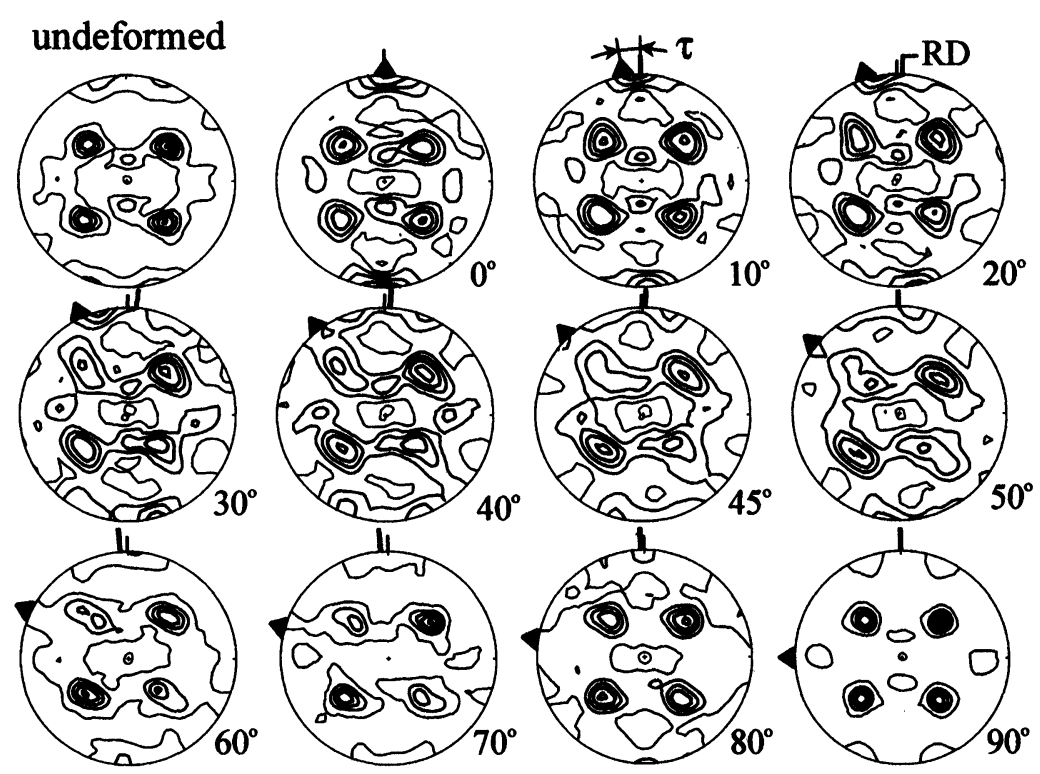

FIGURE 8 (111) pole figures of the starting material, i.e. recrystallized $\mathrm{Al}$ with cube texture and after $20 \%$ elongation under the angle $\tau$ with respect to the original rolling direction. The original rolling direction $\mathrm{RD}$, the stretching direction $\mathrm{SD}$ and the symmetry direction are indicated.

texture coefficients $C_{\lambda}^{\mu n}$ the texture similarity parameter $\varphi^{1,2}(\Delta g)$, Eq. (62), was determined whereby any of the textures was compared with the one deformed in rolling direction $\tau=0^{\circ}$. Since the deformation process does not violate the mirror symmetry in the sheet plane, the actual symmetry of the deformed samples is monoclinic (rather than triclinic). Hence, it is sufficient to plot the similarity parameter only as a function of a rotation $\beta$ about the sheet normal direction. The result is shown in Fig. 9 for two selected cases, i.e. $\tau=0^{\circ}$ and $\tau=20^{\circ}$. The curve for $\tau=0^{\circ}$ compares a texture with itself (rotated through the angle $\beta$ ). It is seen that the minimum at $\beta=0^{\circ}$ goes down to zero (identity) but there are more "fuzzy" similarities at $\beta=90^{\circ}$ and $\beta \pm 45^{\circ}$ corresponding to the orthorhombic and tetragonal components in the sample symmetry already mentioned in Fig. 3. These similarities can still be seen in the sample deformed at $\tau=20^{\circ}$, though much more "fuzzy". The angle $\Delta \beta$ at which the highest similarity is reached, i.e. the minimum of $\varphi^{1,2}(\beta)$ is plotted in Fig. 10. The angle $\Delta \beta$ corresponds to the direction of 


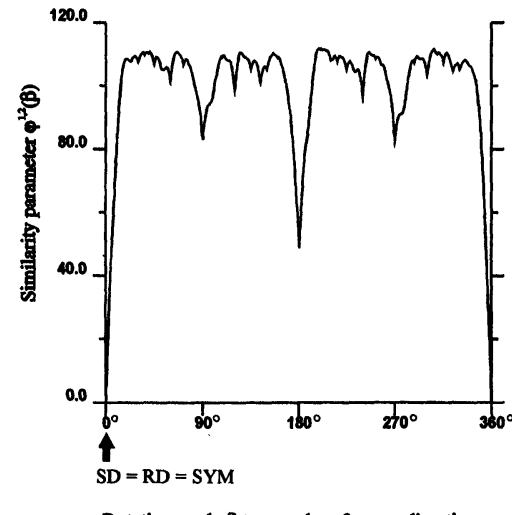

a) Strain direction $\tau=0^{\circ}$

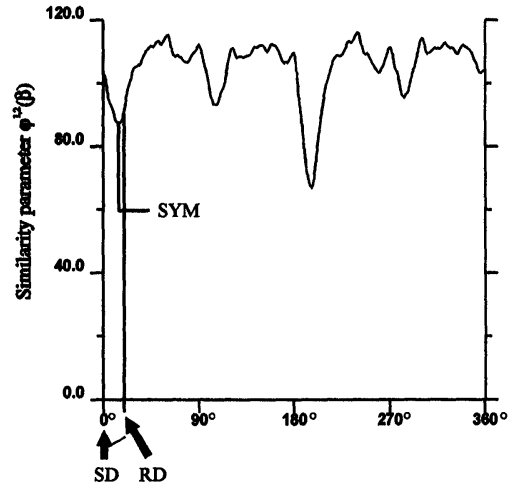

Rotation angle $\beta$ to sample reference direction

b) Strain direction $\tau=20^{\circ}$

FIGURE 9 The texture similarity parameter $\varphi^{1,2}(\Delta g)$ for $\beta=90^{\circ}+\varphi_{1}$ (comparison with the texture deformed at $\tau=0^{\circ}$ ): (a) deformed at $\tau=0^{\circ}$ (self-similarity); (b) deformed at $\tau=20^{\circ}$.

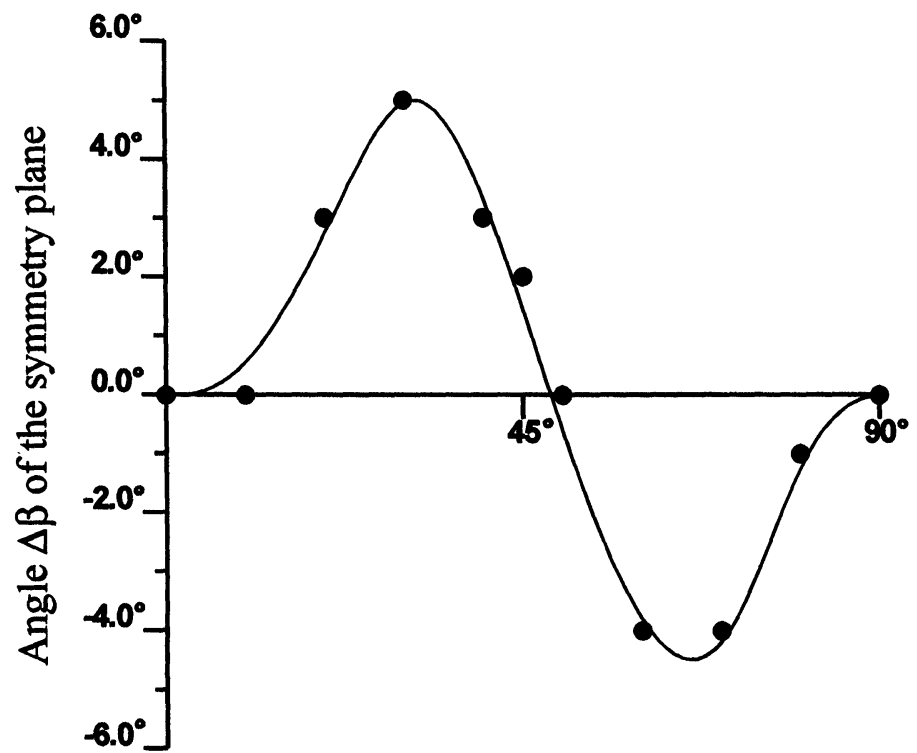

Angle $\tau$ of drawing direction

FIGURE 10 The angle $\Delta \beta$ corresponding to the minimal value of the similarity parameter $\varphi^{1,2}$ of the textures of Fig 8 
the symmetry axes in the deformed samples, Fig. 8. We can also calculate the symmetry parameter $\varphi^{M}(\beta)$ as in Figs. 2-4 for each deformed sample, i.e. each angle $\tau$ separately. It has a minimum value at $\beta_{0}(\tau)$. This minimum value $\varphi_{\min }^{M}(\tau)$ is plotted in Fig. 11. It is seen that the symmetries of the deformed textures are more and more fuzzy the more the angle $\tau$ deviates from the original symmetry axes in $\tau=0^{\circ}$ and $\tau=90^{\circ}$. But also stretching the sample at $\tau=45^{\circ}$ conserves the symmetry (Bunge and Nielsen).

The final example concerns a magnetite ore. From a big piece of this material two samples were taken but the ralative orientation of the sample axes was not specified. The (111) pole figures of them are shown in Fig. 12. Only later on it turned out to be necessary to refer the two textures to the same sample coordinate system. This relationship could be retrieved under the assumption that the two textures from nearby locations of the original big piece were similar enough. Hence, the similarity parameter $\varphi^{1,2}(\Delta g)$, Eq. (62), was calculated in the whole range $\Delta g=\left\{\varphi_{1} \phi \varphi_{2}\right\}$. The result is shown in Fig. 13. It is seen that the similarity parameter has a well-developed minimum which gives the most probable relative orientation of the two-sample coordinate systems.

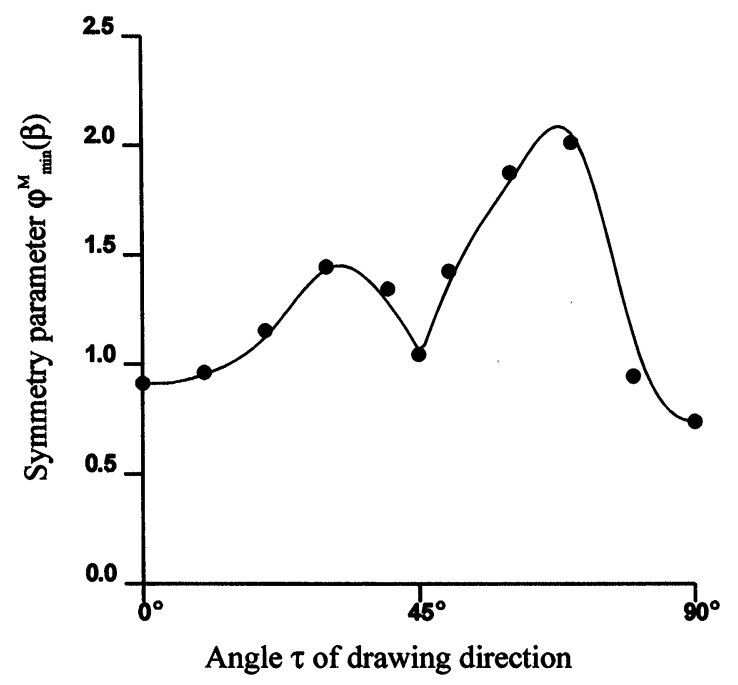

FIGURE 11 Minimal value of the symmetry parameter $\varphi^{M}(\beta)$ as a function of the angle $\tau$ of the drawing direction. 


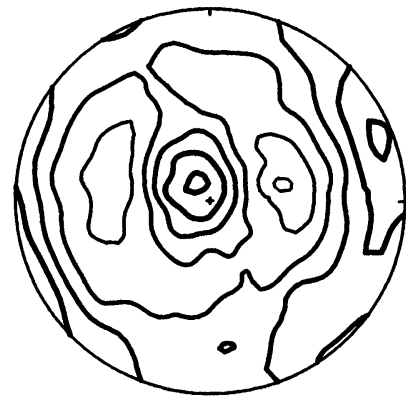

111

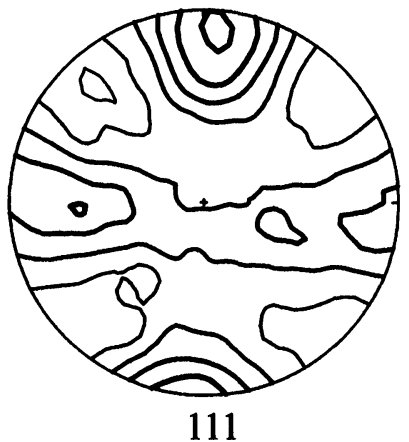

FIGURE 12 (111) pole figures of two samples taken from a magnetite ore sample.

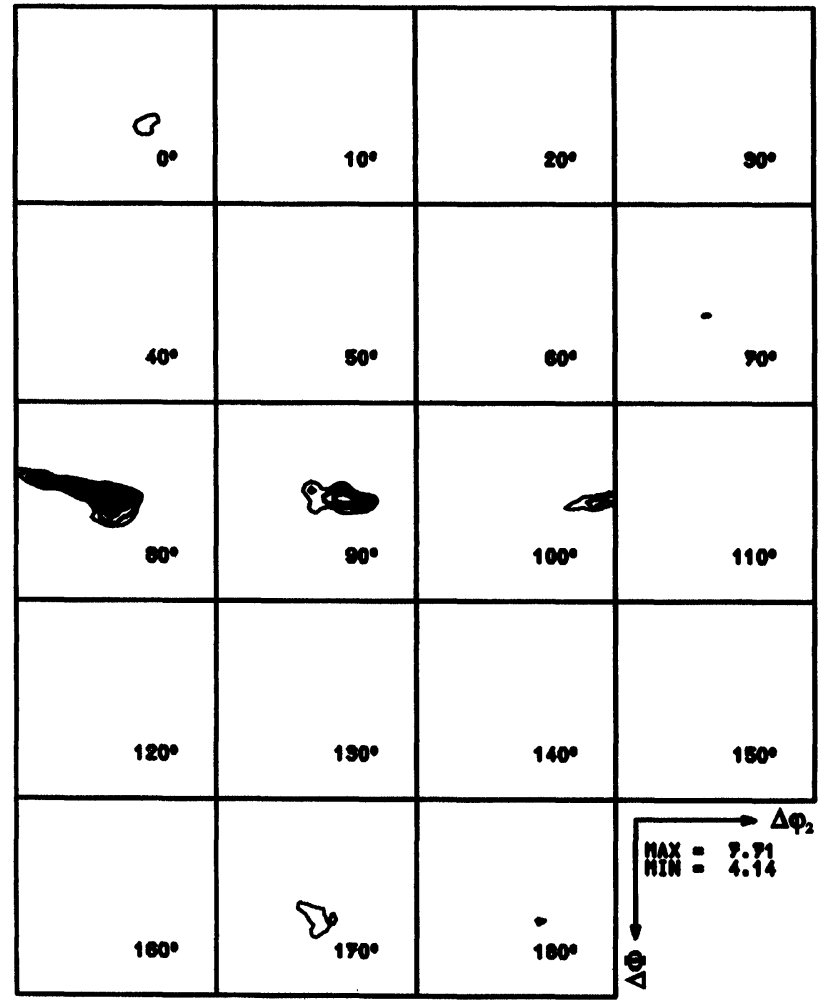

FIGURE 13 Similarity parameter $\varphi^{1,2}(\Delta g)$ in the whole $\Delta g$-space for the two textures of Fig. 12. 


\section{CONCLUSIONS}

The orientation distribution function ODF of the crystallites of polycrystalline materials may exhibit (statistical) symmetries, i.e. sample symmetries, which are the result of corresponding symmetries of the production process. It turns out that these symmetries are often rather "fuzzy" compared with crystal symmetry. It is then necessary to characterize them by a "symmetry degree" which may vary in a wide range from very sharp to rather diffuse symmetries. Also there may be remnants of symmetries from several production steps superposed which do not form a symmetry group. Nevertheless, such generalized "symmetroids" may be successfully used to judge the influences of subsequent production steps which the material has undergone.

Several different symmetry parameters can be defined. If texture analysis is done by the series expansion method (harmonic method) then the most convenient way is to use symmetry parameters based on the texture coefficients $C_{\lambda}^{\mu n}$ which are already provided by routine texture analysis programs.

In several examples it was shown that the symmetry parameters exhibit very sharp minima (cusps) which allow to find the position of symmetry elements in a sample with high precision, e.g. $0.5^{\circ}$. This was successfully applied to determine a misalignment correction for the rolling direction in texture measurement with much higher precision than it was possible without this method.

In a similar way the alignment angles can also be found for the oblique section method of texture measurement.

Symmetry parameters are integrals over the whole orientation distribution function. As such they are rather insensitive to the particular features of the texture. This is particularly helpful in the case of coarse grained materials in which symmetries can still be distinguished even if the texture has great "statistical noise", i.e. it looks "spiky" as in Fig. 4.

The high sensitivity with which symmetry elements can be localized allows to identify small changes of their positions also with high sensitivity. As an example the rotation of the mirror plane after extension of sheet samples in oblique directions was considered. This has been called the "plastic spin" in terms of plastomechanics (see e.g. Dafalias, 1984; Raniecki and Mróz, 1989). 
At the same time it is also seen that the "plastic spin" describes only part of the texture changes after oblique extension. Also the degree of symmetry is increasingly modified the more the extension direction deviates from the original symmetry directions. This is important, for instance, in deep drawing where the direction of extension varies continuously around the circumference of a drawn cup, thus leading to cup-earing.

Besides the symmetry parameters also similarity parameters can be defined which compare two textures with each other. Also similarity parameters are a useful tool. This was shown, for instance, in two geological samples taken from neighbouring positions. On the one hand, it showed that neighbouring samples have similar textures, and, on the other hand, it allowed to find the corresponding sample axes with high accuracy. With the help of the so defined symmetry parameters as well as similarity parameters, formerly only qualitatively considered features of textures, e.g. "fuzzy symmetries" or "similarity" can now be defined on a quantitative basis. This may have many practical applications as was shown here only in a few selected examples.

\section{Acknowledgement}

The authors gratefully acknowledge sponsoring by the German Research Foundation DFG.

\section{References}

Bunge, H.J. (1982) Texture Analysis in "Materials Science. Butterworths Publ. London, 2nd Ed. Cuvillier Verlag, Göttingen, 1993.

Welch, P.I. (1980) Techniques for the determination of complete pole figures using composite specimens. Texture of Crystalline Solids 4, 99-110.

Dafalias, Y.F. (1984) The plastic spin concept and a simple illustration of its role in finite plastic transformations. Mech. of Mat. 3, 223.

Raniecki, B. and Mróz, Z. (1989) On the strain-induced anisotropy and texture in rigidplastic solids. In: Inelastic Solids and Structures (Eds., Kleiber, M. and König, A.) Pineridge Press, Swansea.

Nielsen, I. and Bunge, H.J. (1996) Symmetry elements in textures. Proc ICOTOM-11. Xian (China), 156.

Bunge, H.J. and Nielsen, I., Experimental determination of plastic spin (in preparation). 